LAWRENCE LIVERMORE NATIONAL LABORATORY
NIF final optics system: frequency conversion and beam conditioning

P. Wegner, J. Auerbach, T. Biesiada, S. Dixit, J. Lawson, J. Menapace, T. Parham, D. Swift, P. Whitman, and W. Williams

January 28, 2004

SPIE Photonics West, San Jose, California, January 24-29, 2004 
This document was prepared as an account of work sponsored by an agency of the United States Government. Neither the United States Government nor the University of California nor any of their employees, makes any warranty, express or implied, or assumes any legal liability or responsibility for the accuracy, completeness, or usefulness of any information, apparatus, product, or process disclosed, or represents that its use would not infringe privately owned rights. Reference herein to any specific commercial product, process, or service by trade name, trademark, manufacturer, or otherwise, does not necessarily constitute or imply its endorsement, recommendation, or favoring by the United States Government or the University of California. The views and opinions of authors expressed herein do not necessarily state or reflect those of the United States Government or the University of California, and shall not be used for advertising or product endorsement purposes. 


\title{
NIF final optics system: frequency conversion and beam conditioning*
}

\author{
P. Wegner, J. Auerbach, T. Biesiada, S. Dixit, J. Lawson, J. Menapace, T. Parham, \\ D. Swift, P. Whitman, W. Williams \\ Lawrence Livermore National Laboratory, PO Box 808, Livermore, CA, USA 94550
}

*Work performed under the auspices of the U.S. Department of Energy by the University of California, Lawrence Livermore National Laboratory under Contract No W-7405-Eng-48.

\begin{abstract}
Installation and commissioning of the first of forty-eight Final Optics Assemblies on the National Ignition Facility was completed this past year. This activity culminated in the delivery of first light to a target. The final optics design is described and selected results from first-article commissioning and performance tests are presented.
\end{abstract}

Keywords: NIF, frequency conversion, diffractive optics, solid-state lasers, ICF

\section{INTRODUCTION}

The National Ignition Facility (NIF) is a megajoule-class solid-state laser and targeting facility under construction by the Department of Energy for stockpile stewardship and inertial confinement fusion (ICF) research [1]. The amplified 1.053- $\mu \mathrm{m}$ beams from the Nd:glass driver are transported in $2 \mathrm{x} 2$ quads to the $10-\mathrm{m}$ diameter target chamber where 48 final optics assemblies (FOAs) frequency convert each quad to the third harmonic and focus them onto the target. Each FOA consists of a fixed system interface to the target chamber, and four integrated optics

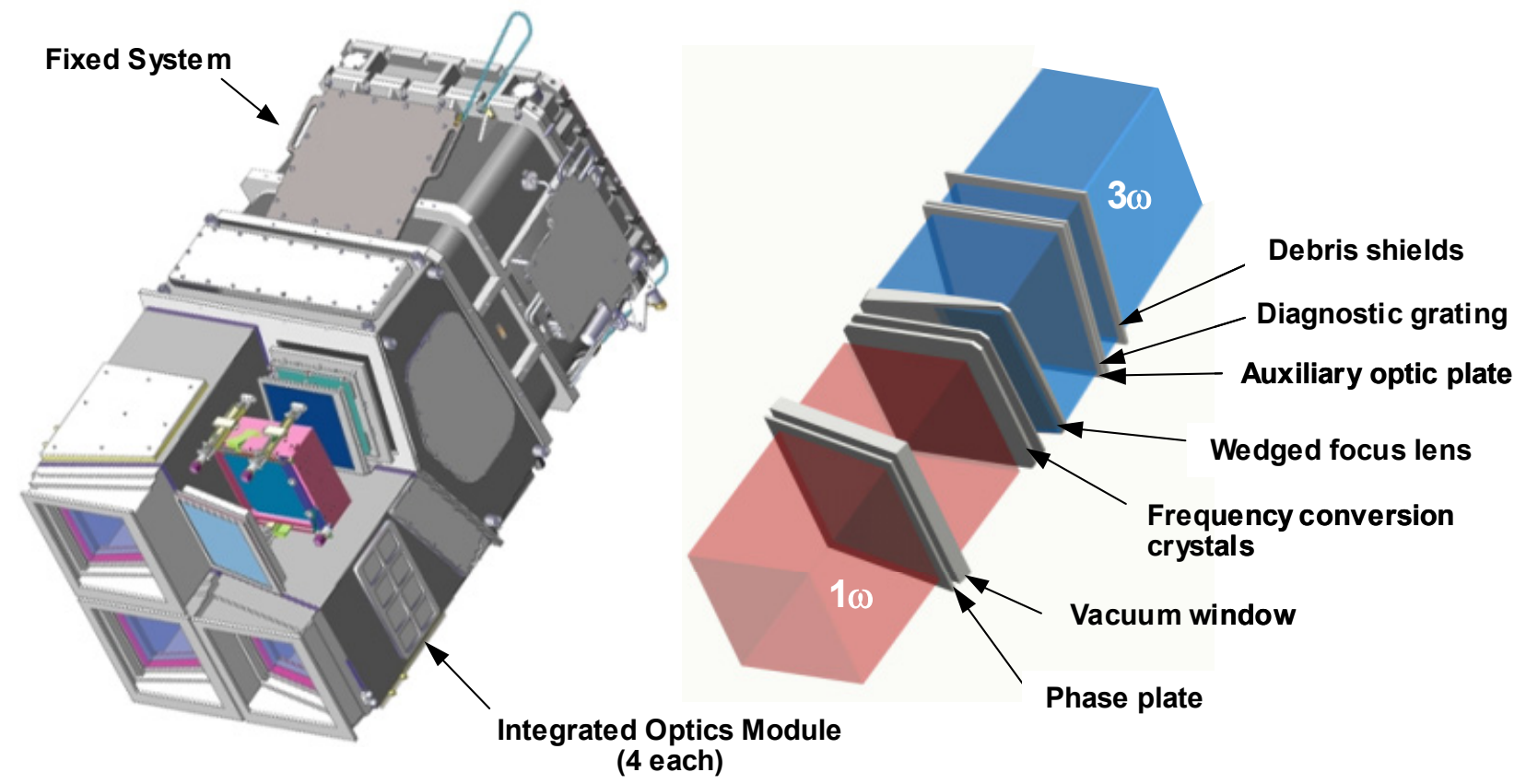

Figure 1. The FOA is comprised of a fixed system that mounts on the target chamber and four LRUs containing the final optics for each beam. The clear aperture is $40 \mathrm{~cm}$. 
modules (IOMs) that house the final optics. These precision opto-mechanical systems combine a number of critical functions into a single compact package: frequency conversion, focusing, color separation, diagnostic beam sampling, vacuum isolation and debris shielding. The frequency converter is a cascade sum-frequency generation design consisting of a $1.1-\mathrm{cm}$ thick Type-I KDP doubler and a $0.9-\mathrm{cm}$ thick Type-II deuterated-KDP tripler, optimized to achieve $>80 \%$ peak power conversion efficiency to the third harmonic at a $1 \omega$ drive irradiance of $3 \mathrm{GW} / \mathrm{cm}^{2}[2]$. The 40-cm aperture crystals are mounted with full-perimeter support in a precision machined aluminum cell that maintains their relative orientation to better than $10 \mu \mathrm{rad}$ and flatness to better than $10 \mu \mathrm{m}$. The cell also houses a fused-silica final focus lens, which is of wedged design to provide color separation at the target via lateral dispersion. As beam pointing is adjusted into the chamber for different targeting scenarios the cell is tipped as a unit to maintain absolute angular alignment of the lens and crystals. Approximately $0.2 \%$ of the frequency converted beam energy exiting the lens is directed to a $3 \omega$ diagnostics sensor by means of a fused-silica grating, which samples the full-aperture beam with $\sim 5 \%$ rms spatial uniformity. The components inside the IOM are operated at a pressure of 10 torr with a 1-SLPM purge of clean dry air, separated from the argon environment of the beam transport system by a $1 \omega$ pressure window, and the hard vacuum of the target chamber by a 1-cm thick gas-seal debris shield. The $1 \omega$ window is preferred over a $3 \omega$ window for safety reasons, and the low-pressure air purge provides improved AR coating stability and increased optics damage resistance over operation in vacuum.

The FOA design represents an integrated solution to a large number of performance, operational, and environmental requirements that are too extensive to address in detail here. The following section instead provides a general system overview, emphasizing design aspects that have proven particularly challenging. Subsequent sections describe in more detail the selected functions of frequency conversion and beam conditioning.

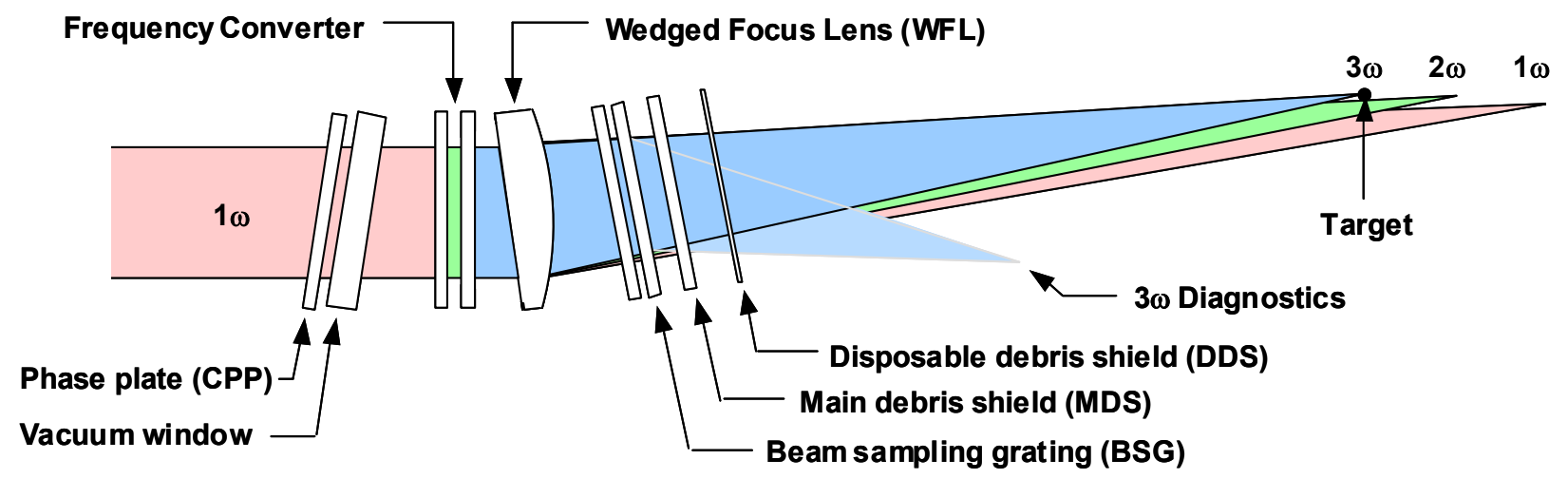

Figure 2. Schematic of the optical configuration of the FOA

\section{DESIGN OVERVIEW}

The FOA optical configuration, shown schematically in Figure 2, is designed to deliver the required functionality with the fewest possible components. The $1.053-\mu \mathrm{m}$ beam from the main laser enters the package through a $4.3-\mathrm{cm}$ fused silica vacuum window, sized so that the internal stresses in the window are kept below 500 psi for safety. Inside the package, the beam passes through a frequency converter (described in the next section) and a fusedsilica lens that focuses the resulting $3 \omega$ light onto the target. The lens is a reversed plano-convex aspheric design of 7.7- $\mathrm{m}$ focal length, with a wedge applied to the first surface to steer the beam toward the center of the quad in one axis (see Figure 3). This use of the wedge allows the four beam apertures in each FOA to be arranged in close-packed $2 \times 2$ arrays without clocking. It also serves the important function of angularly dispersing the unconverted $1 \omega$ and $2 \omega$ light away from the target (Figure 4). One drawback of the reversed lens design is that it requires the second surface of the lens to be coaxial with the $3 \omega$ chief ray to within $\sim 400 \mu \mathrm{rad}$ to maintain transmitted wavefront errors below $\lambda / 4$. The required alignment is maintained by precision mounting the lens in a cell along with the frequency conversion crystals, and actively aligning the cell to the beam as described in the next section. 

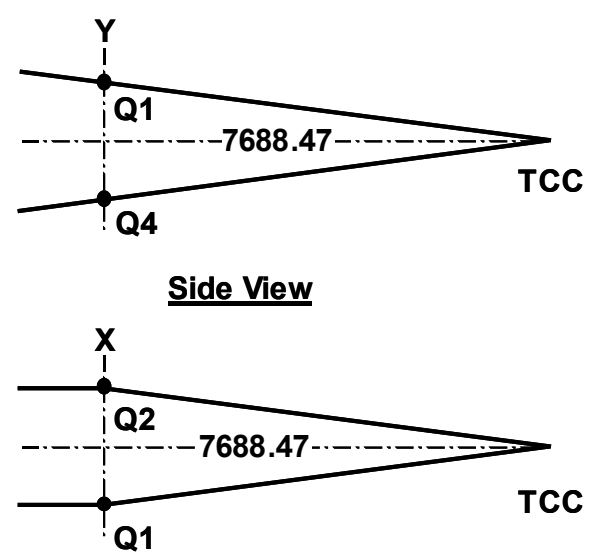

$\underline{\text { Top View }}$

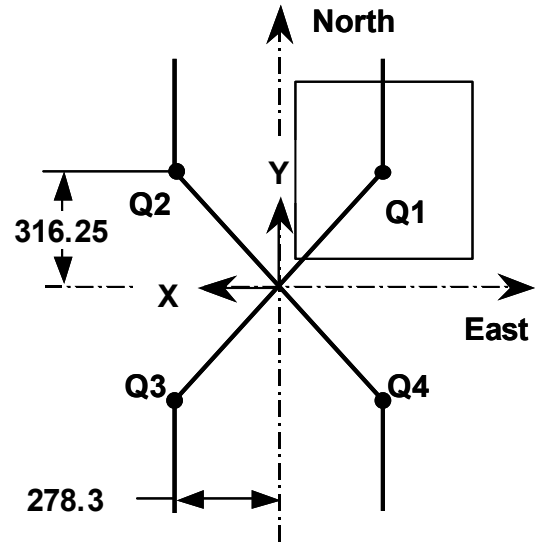

View Toward TCC

Figure 3. Beampath geometry at the FOA. The wedged lens is used to steer the beams toward target chamber center (TCC) in the $\mathrm{x}-\mathrm{z}$ plane

The remaining components in the package consist of 1-cm thick plates that can be inserted and removed from the assembly as required via cassette. Focal spot conditioning, described further in Section 4, is achieved by means of a continuous-contour phase plate (CPP) inserted upstream of the vacuum window and an optional polarization scrambler inserted in the first cassette slot downstream of the lens. The second slot downstream of the lens contains a fused silica plate with a shallow ( 20-nm deep) grating etched into the second surface. The grating directs a weak sample of the $3 \omega$ beam to a diagnostic package located in the fixed system portion of the FOA. The last slot in the package is reserved for the main debris shield. The MDS is fabricated from fused silica and resides in a special bezel that once inserted, cams up against a mating surface in the IOM to form a gasket seal isolating the interior of the IOM from the target chamber. The MDS is itself protected from target debris by a thin ( 1-mm thick) disposable debris shield (DDS) fabricated from inexpensive boro-silicate float glass. The DDS is designed to be used for one to ten shots, depending on the experiment, before it requires replacement.

The optical configuration satisfies a number of challenging design constraints. Stray light management, for example, is an important consideration that has profoundly impacted the design. The energy lost to surface reflections inside the FOA alone exceeds the output energy of many kJ-class lasers; this energy must be safely managed and dissipated inside the package. To help accomplish this, most of the plates are tilted to direct bathing ghosts towards the outside walls of the FOA. There, panels of architectural glass absorb the energy and transfer the resulting heat to walls of the IOM where it is dissipated by means of conduction to passive water cooling panels and convection to the temperature-controlled air inside the target bay. The final optics cell (FOC) containing the crystals and the lens is lined with polished NG-glass to intercept fluences that are generally higher than those on the walls of the IOM. Focusing ghosts are another concern that place severe constraints on component placement. For example, a third-order ghost that focuses upstream of the target chamber vacuum window sets the maximum

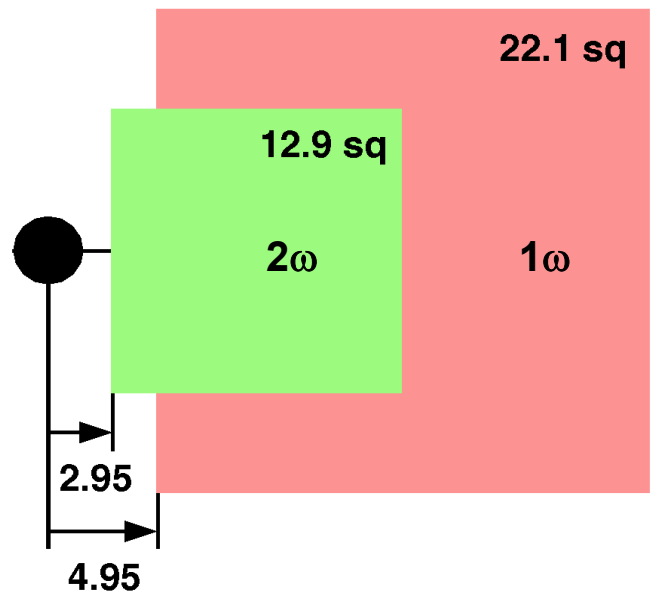

Figure 4. Location of unconverted light for a single beam focused at the target. Dimensions are in $\mathrm{mm}$. 
allowable spacing between the lens and the conversion crystals and limits the range of travel that can be used to adjust focus about target chamber center.

Total glass thickness is an important design consideration that ultimately limits the power densities at which the system can be operated. In the perturbation regime, low-level noise power in the beam is expected to grow as $\mathrm{e}^{2 B}$ where $B$ is the well-known B-integral. Increasing glass thickness results in higher $B$ and a decrease in focusable power on target. Numerical beam propagation studies of the FOA using high-resolution patches demonstrate how the fractional power at large angles increases with laser intensity due to nonlinear refractive index (Figure 5). Noise in the FOA arises predominantly from small-scale phase errors associated with optical fabrication and of these, the dominant contributors are the diamond-turned surfaces of the frequency-conversion crystals. The figure demonstrates the importance of maintaining tight tolerances on the crystal finish, particularly in the portion of the PSD2 band covering spatial scale lengths between 125 and $500 \mu \mathrm{m}$ (previously referred to as PSD3 [3]). These studies were used to set the current specification for this band of 2-nm rms. At this level, a combination of patch and full-aperture simulations predict that the optical configuration should be able to meet a high-brightness goal for the facility of $500 \mathrm{TW}$ ( $67 \%$ of the power) inside a $250-\mu \mathrm{m}$ diameter spot.

A final example of the type of design constraint encountered in the FOA is cleanliness-- an over-riding concern that has impacted all aspects of the design, assembly and operation of this system. Specifications for particulate cleanliness inside the IOM are Level 100 for the assembled hardware and Level 50 for the assembled optics, requiring extreme care in the material selections and design of the internal mechanical systems and related optical assembly and installation hardware. Strict adherence to established cleaning procedures and Level $4+$ protocols during assembly is also required. The difficulty of meeting these requirements in a complex mechanical system the size of the IOM cannot be understated. Nonetheless, compliance has been achieved and verified to the extent permitted by wafer tests and bright-light inspections. The specification of $\mathrm{A} / 10$ for organic cleanliness is also very stringent, as dictated by the porous sol-gel anti-reflection coatings that are used on the optics. Figure 6 demonstrates the great strides that have been made in improving the chemical resistance of these coatings to organic contamination, relative to the time of Beamlet when AR-coating degradation in vacuum was recognized as a significant factor in the frequency converter performance $[2,4]$. Pore filling and

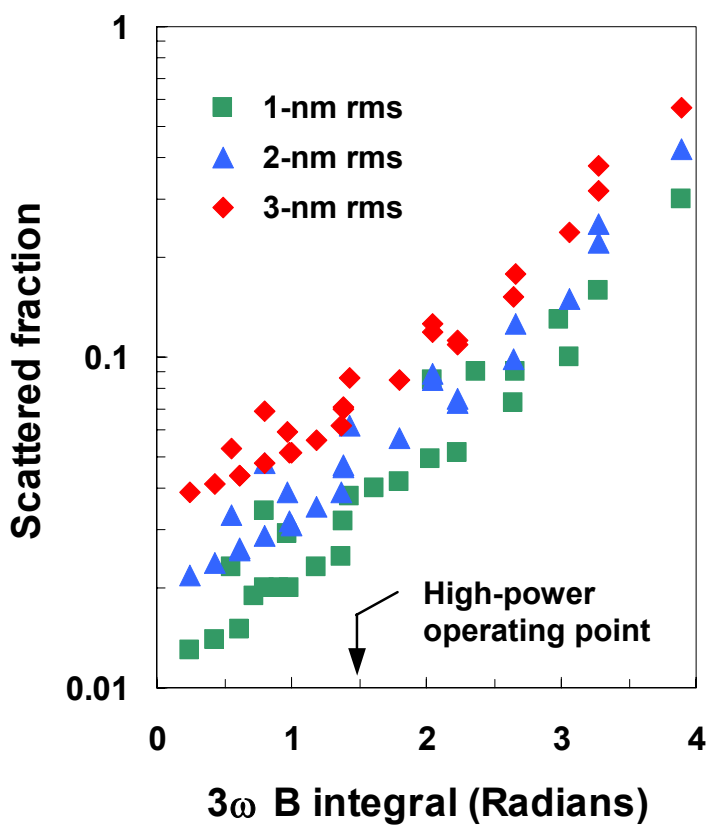

Figure 5. Calculations of beam propagation through the FOA showing the increase in wide-angle scattering $(>45 \mu \mathrm{rad})$ that occurs at high B-integral, and its dependence on crystal surface finish quality in the PSD2 band

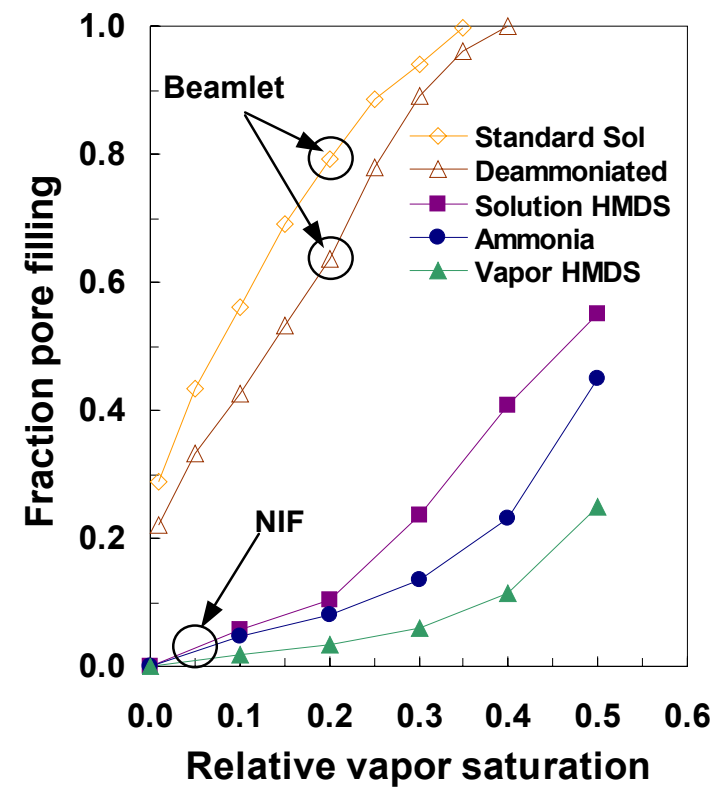

Figure 6. Absorption isotherms for different types of solgel anti-reflection coating, showing the difference between the Beamlet-era coatings, and vapor-HMDS treated coatings used on NIF. High levels of pore filling degrade the AR properties of the coatings. 
the resulting increase in coating reflectivity is further controlled in the design by eliminating organic contamination sources inside the IOM and operating the internal optics with a filtered dry-air purge of 1 SLPM at a static pressure of 10 torr. Long-term monitoring of the internal IOM environment with a sol-gel-coated surface-acoustic wave transducer has verified that this design strategy successfully keeps pore-filling below 5\%, and the transmission through the AR coatings optimized to within a few tenths of a percent.
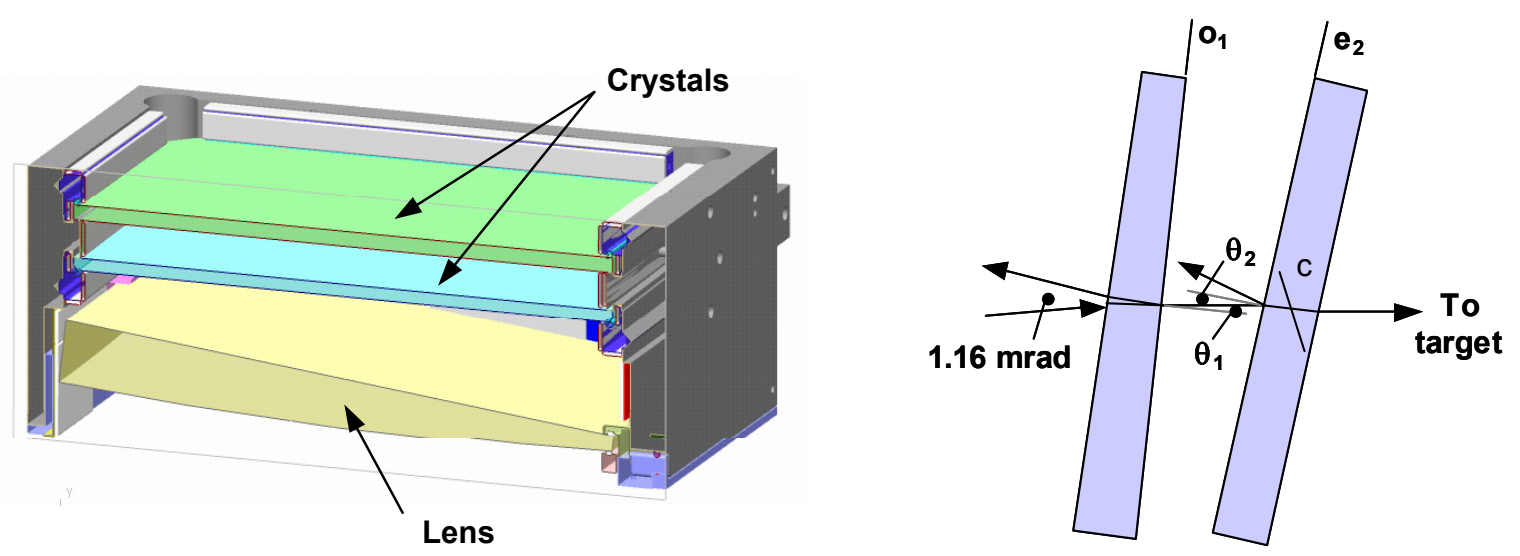

Figure 7. Cut-away view of the final optics cell showing the frequency conversion crystals and the final focus lens. The tilt of the crystals relative to the beam axis are shown schematically on the right. The external angle of incidence at the output of the doubler $\left(\theta_{1}\right)$ and the input of the tripler $\left(\theta_{2}\right)$ are 0.580 and $10.580 \mathrm{mrad}$, respectively. The orientation of the tripler extraordinary axis is indicated. The doubler extraordinary axis is into the plane of the figure.

\section{FREQUENCY CONVERSION}

The design and engineering of the NIF frequency converter has been described previously [2]. Two crystalsan 11-mm type-I KDP doubling crystals and a 9-mm type-II dKDP tripling crystal ( $\sim 0 \%$ deuterated)- are mounted together in a single aluminum structure called the final optics cell as shown in Figure 7. The output surface of the doubler serves as the alignment reference for the cell. Precision-machined lands register this surface to the input surface of the tripler with better than 10- $\mu$ rad accuracy. The doubler is designed and cut to be operated with its output surface at normal incidence to the beam in its sensitive (e) direction and $0.58-\mathrm{mrad}$ (external) angle of incidence in its insensitive (o) direction. The tripler is designed to be operated at normal incidence in its insensitive direction and $10.58-\mathrm{mrad}$ angle of incidence in its sensitive direction. When the cell is correctly oriented, the reflection from the doubler focuses at a location in the transport spatial filter (TSF) pinhole plane that is offset laterally from the pinhole by $1.16 \mathrm{mrad}$. A slight wedge applied to the doubler along its insensitive axis separates its two surface returns in the TSF, allowing the $2^{\text {nd }}$ surface reflection to be clearly identified and aligned. Bevels applied to the edges of the crystals limit the gain for transverse-stimulated Raman scattering to a single pass [5].

The crystals are held against the mounting lands with a full-perimeter compliant clamp of 1 to $2 \mathrm{lbs} /$ linear inch to help counteract the gravity-sag that would otherwise occur with more-traditional three-point or corner mounting schemes. The challenge for this design is maintaining the flatness of the mounted crystals. Figure 8 shows interferograms of mounted crystals obtained as part of the metrology conducted for an FOC representative of the cells assembled for NIF. The impact of the observed figure error on converter alignment has been evaluated numerically by adding measured crystal figures similar to those in the figure to gravity-sag distortions modeled with finite-element analysis and calculating the resulting shift in the alignment spot centroid observed in the TSF. The result, shown in Figure 9, predicts a small centroid shift of on the order of a few $\mu \mathrm{rad}$ internal angle. The shift is caused by clipping of the crystal return at the entrance to the TSF, made visible at the pinhole plane by the defocus induced by the gravity sag. The simulation is seen to be in excellent agreement with crystal returns measured on the system, giving high confidence that the mounting scheme is performing as expected. 

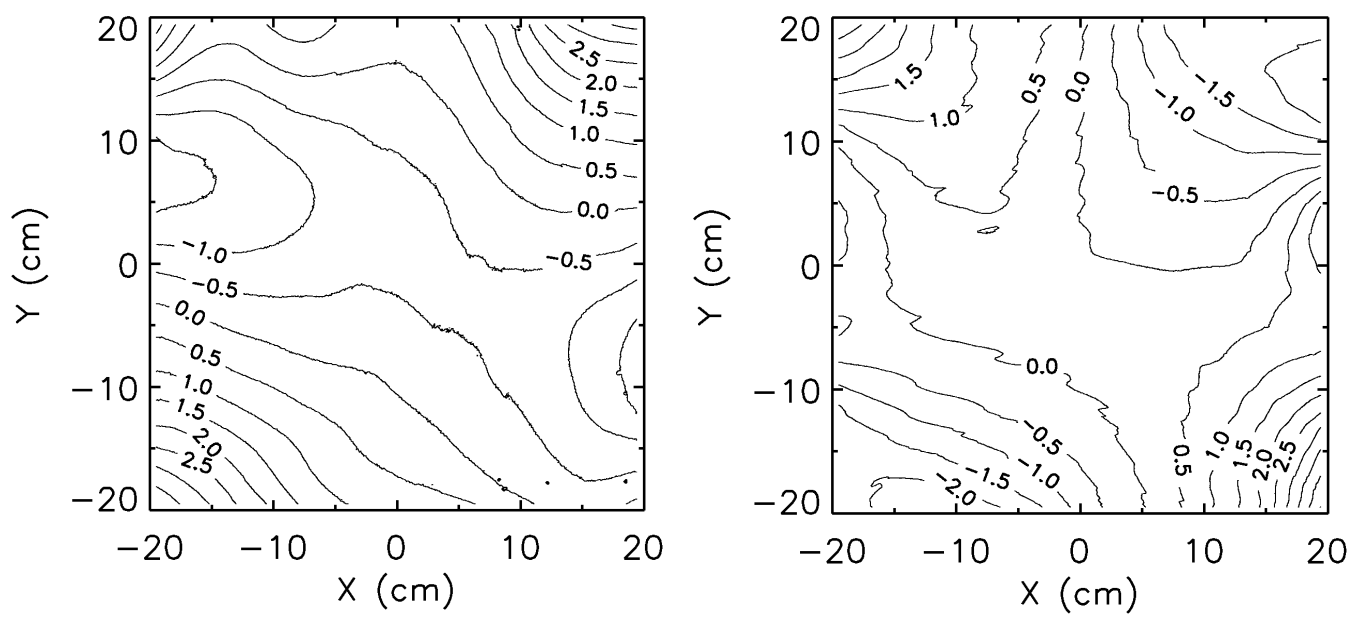

Figure 8. Measured surface figures of a doubler (left) and a tripler (right) mounted in a NIF FOC. The data was taken with the crystals oriented vertically with respect to gravity. Contour interval is $0.5 \mu \mathrm{m}$.

TSF

FOC

TCC
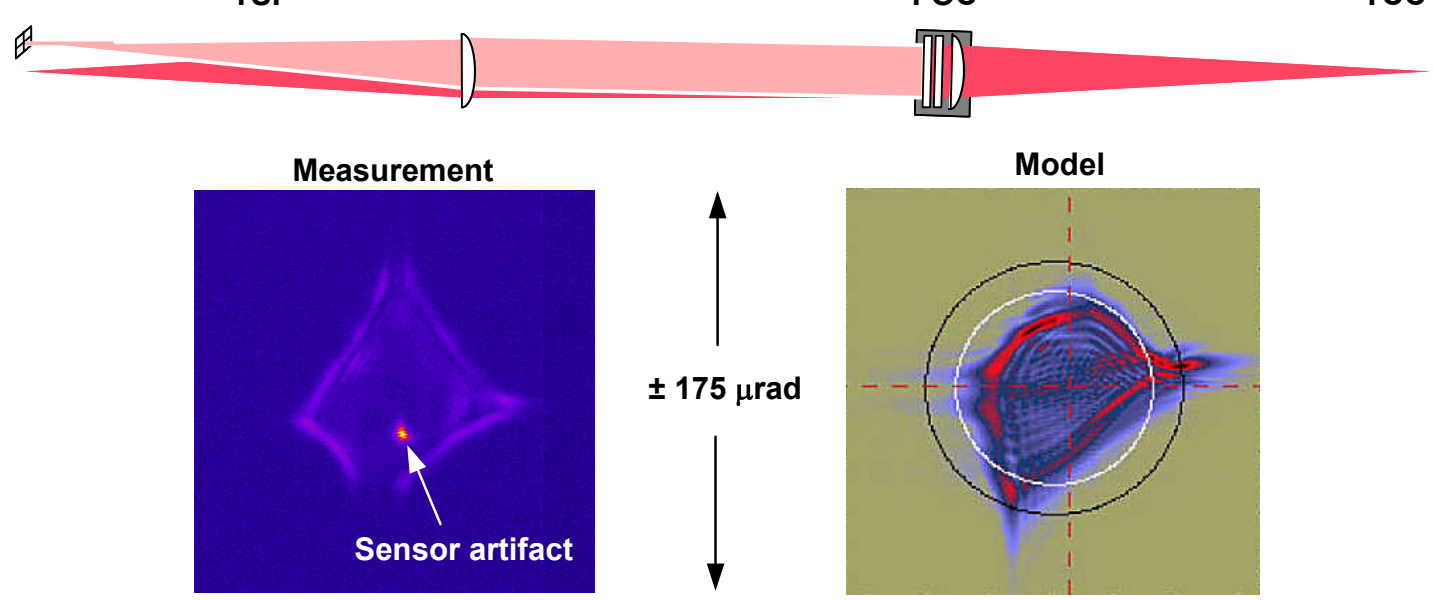

Figure 9. FOC alignment is achieved using the TSF as an autocollimator to view the position of the focused doubler return relative to the main laser pass-4 pinhole. Gravity sag induces curvature on the crystal's reflected wavefront, which shifts the focus in $\mathrm{z}$ relative to pinhole plane causing the spot to appear defocused. Current alignment algorithms can accurately position the centroid of this spot to better than $5 \mu \mathrm{rad}$.

Frequency conversion performance of the FOA has been tested and verified as part of the NIF Early Light commissioning campaigns described elsewhere [6]. Figure 10 summarize the results of two of these campaigns conducted using the Precision Diagnostic System (PDS). The PDS is a reincarnation of the Beamlet Focal Plane Diagnostic that is now installed in NIF Switchyard 2 [7]. It has a comprehensive suite of diagnostics, including calorimetry at all three wavelengths, that allow a far-more complete evaluation of the converter than can be achieved on the target chamber. The first plot in the figure shows the $3 \omega$ energy produced on a single beam as a function of input $1 \omega$ energy for 3.5-ns square pulses. The measurements are compared against theoretical curves produced by a plane-wave model that uses design values for component transmissions, and measured values for the $1 \omega$ beam area and pulse duration The results of full 4-D simulations are also shown for comparison. The second plot shows the $2 \omega$ performance of the FOA as measured with 5-ns square pulses. This data was obtained using a modified FOA configuration in which the tripler was removed from the FOC, the lens and downstream optics were AR-coated for $2 \omega$, 

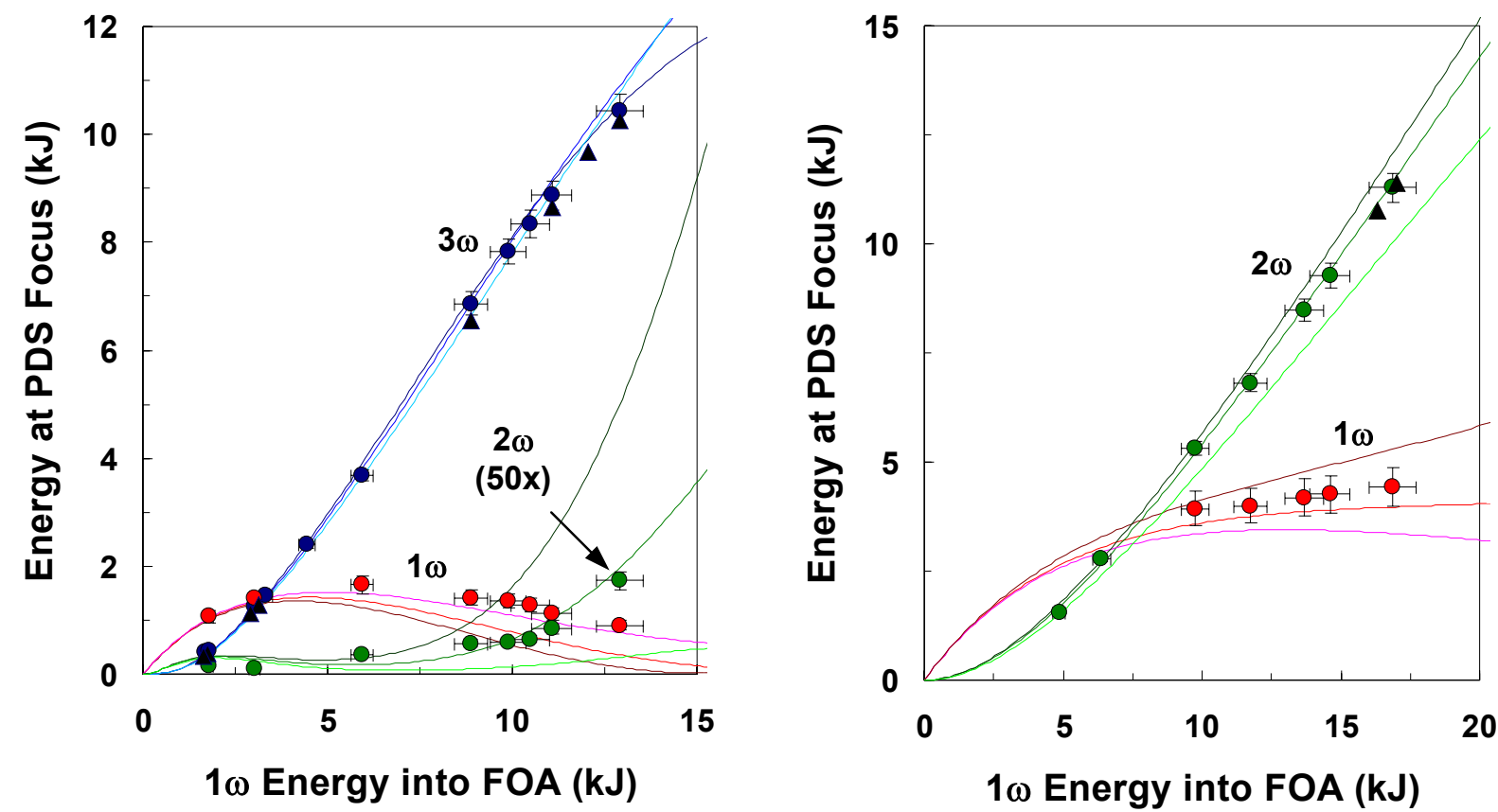

Figure 10. Measured frequency conversion performance to the second and third harmonic. Measured energies of the residual harmonic wavelengths are also plotted. $3 \omega$ theory curves are shown for doubler detunings of $220 \pm 30 \mu \mathrm{rad}$ (internal angle), assuming 30- $\mu \mathrm{rad}$ detuning for the tripler. $2 \omega$ theory curves are shown for doubler detunings of 0,100 and 200 rad. Results of full 4-D simulation are denoted by the triangles.

and the FOC tilted to move the doubler away from the 220- $\mu$ rad detuning required for efficient tripling back towards the zero detuning required for maximum second-harmonic generation. Unfortunately, the range of crystal tilt is limited by the size of the beam dump which captures the reflections in the TSF, with the result that the tests had to be conducted with a residual doubler tuning error of $100 \mu \mathrm{rad}$ (internal angle). Accordingly, the $2 \omega$ results are observed to fall exactly on the 100- $\mu \mathrm{rad}$ theory curve. The agreement of the $3 \omega$ data with theory is also quite remarkable up to the maximum fluences tested. It should be noted that the upper data points in these plots represent world records for energy produced in a single laser aperture at these wavelengths.

\section{BEAM CONDITIONING}

The FOA is designed to accommodate a number of beam conditioning techniques for smoothing the focal spot profile on target, including phase plates, polarization scramblers, and smoothing by spectral dispersion (SSD). Phase plates are an established technology for transforming aberrated laser focal spots into comparatively homogeneous speckle distributions of various shapes and sizes [8]. The phase plates used on NIF are of the continuous (CPP) variety which have smooth profiles to avoid the high levels of near-field intensity modulation associated with the discrete phase jumps of conventional RPPs [9]. Modeling has shown that for spot sizes up to a few mm, CPPs can be installed in the $1 \omega$ section of the laser immediately upstream of the FOA vacuum window without significantly impacting the frequency conversion efficiency or the near-field modulation of the $3 \omega$ beam. The calculations, shown in Figure 12, were performed for an elliptical spot of 2:1 aspect ratio, oriented with the long axis of the ellipse along the sensitive axis of the tripler. The results show that for 1-mm spots, the reduction in conversion efficiency is less than $0.5 \%$ at $3 \mathrm{GW} / \mathrm{cm}^{2}$ and the increase in $3 \omega$ beam contrast is negligible. At $3 \mathrm{~mm}$, the reduction in conversion efficiency has reached $4.5 \%$ and the increase in beam contrast is beginning to be noticeable. Experiments requiring spot sizes larger than $\sim 3 \mathrm{~mm}$ will require careful evaluation to determine whether the CPP should be deployed in the $1 \omega$ or $3 \omega$ section of the FOA. 

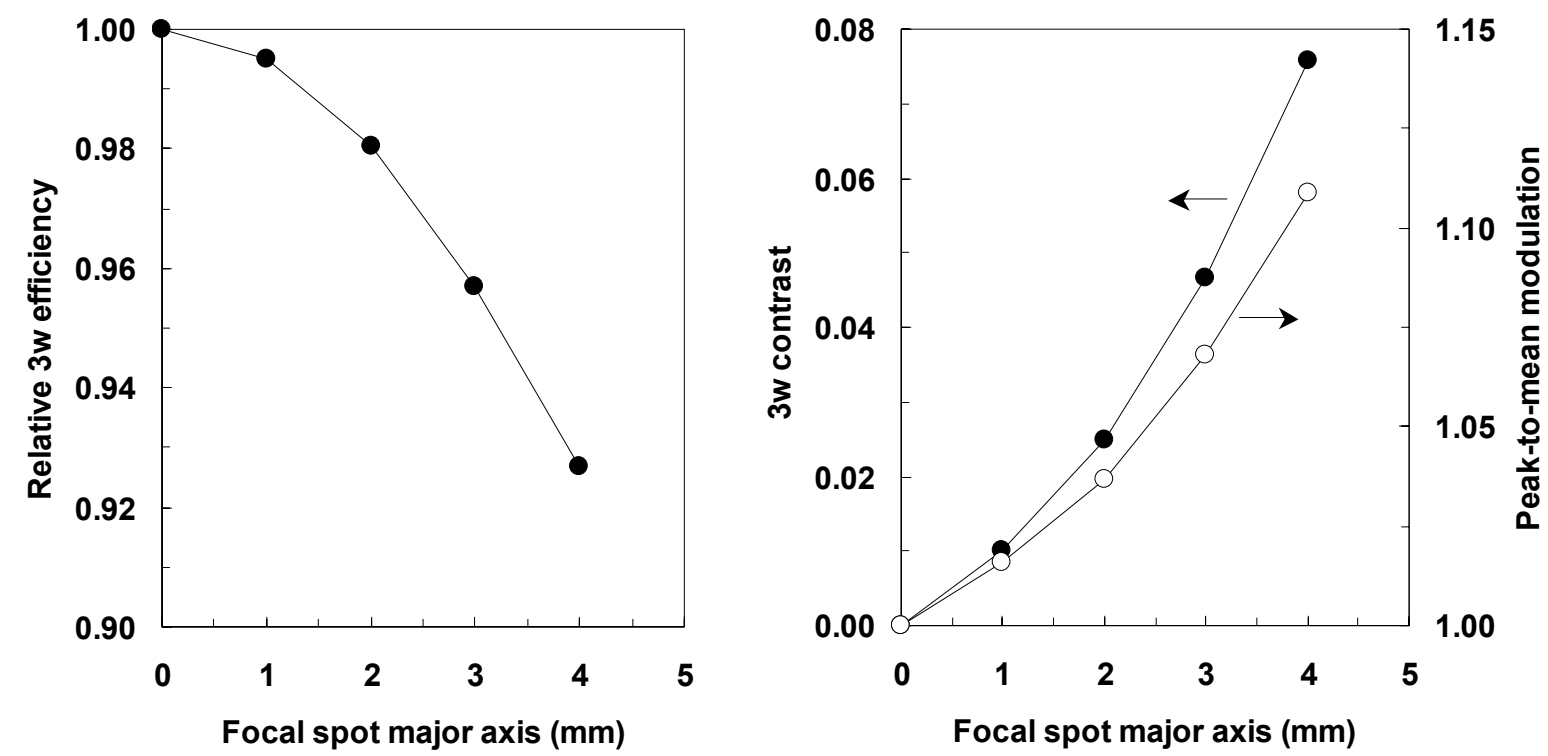

Figure 11. Calculations demonstrating impact of $1 \omega$ CPP spot size on $3 \omega$ conversion efficiency and fluence modulation.

Full-aperture CPPs are in the process of being fabricated for use in NIF experiments. The fabrication method relies on programmable magneto-rheological finishing (MRF) technology to polish the desired phase profile into the fused silica substrate of the CPP [10]. The phase profile for these plates is designed to produce a super-gaussian flattop speckle distribution of 1-mm diameter as shown in Figure 12a. Initial results have exhibited measured phase profiles within $\sim 25 \mathrm{~nm}$ rms of design that produce a calculated focal spot close to the desired shape (Figure 12b). Development is continuing with the goal of testing full-aperture plates on NIF this year. In the meantime, a limited number of beam-conditioning experiments have been conducted with sub-scale 0.5 -mm CPPs installed in the NIF front end. These experiments have utilized up to $60 \mathrm{GHz}$ of $1 \omega$ bandwidth and have produced up to $4 \mathrm{~kJ}$ of $3 \omega$ energy in a conditioned focus both with and without SSD. A more detailed discussion of those experiments is beyond the scope of this paper.
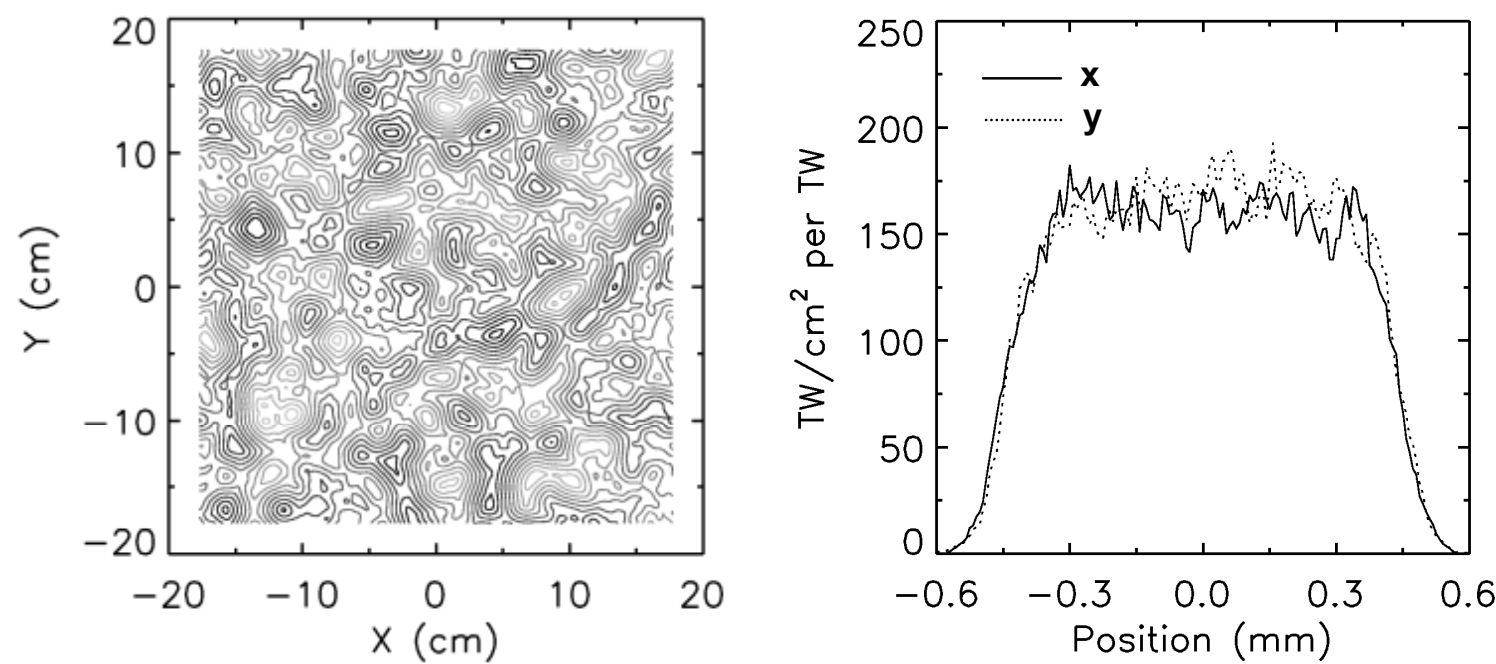

Figure 12a. Design phase profile and focal spot profile for the full-aperture 1-mm CPP. Phase ranges between -2.98 and +2.92 waves $(3 \omega)$ with contours at $0.5 \mathrm{n}$ waves, $-5 \leq \mathrm{n} \leq 5$. Speckle has been smoothed with a $50-\mu \mathrm{m}$ boxcar for the linescans. 

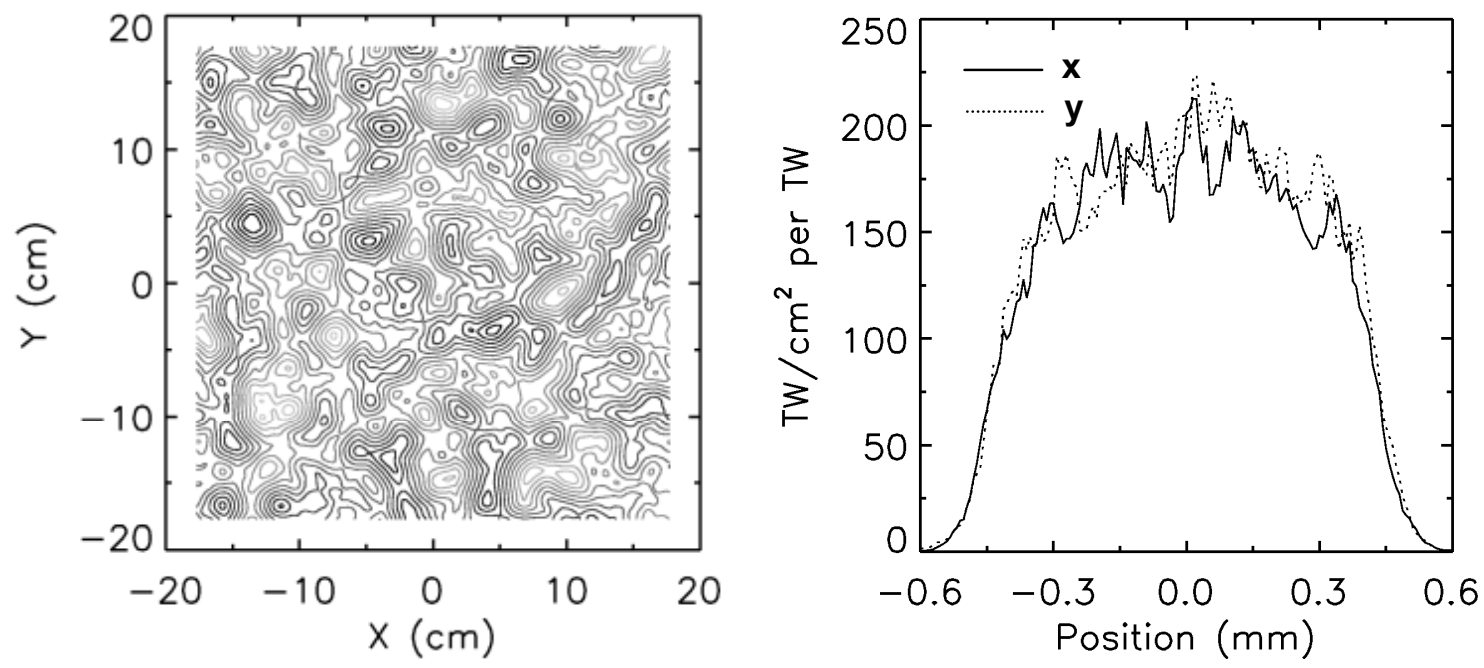

Figure 12b. Measured phase profile and the calculated focal spot profile for the prototype fullaperture 1-mm CPP. Phase ranges between -2.93 and +2.95 waves $(3 \omega)$ with contours as defined in Figure 13a. Speckle has been smoothed with a $50-\mu \mathrm{m}$ boxcar average for the linescans

Polarization smoothing is used to reduce the speckle contrast on target by overlaying uncorrelated speckle patterns of orthogonal polarizations [11]. Established techniques rely on a birefringent optic to split the near-field beam equally into two orthogonally-polarized components, and apply a pointing difference between these components that is sufficient to shift their focal spots by one or more speckle correlation lengths. The polarization smoothing scheme for NIF differs from previously implemented designs in that it is required to operate in a converging beam. The design, described elsewhere [12], consists of a 1-cm thick plane-parallel KDP plate cut from the boule as shown in Figure 13. It produces a 40-60 polarization split in the near-field and a 30- $\mu \mathrm{m}$ offset in the focal spots for the two orthogonal polarizations at the target. Prototypes are in the process of being fabricated for testing on the laser this year.

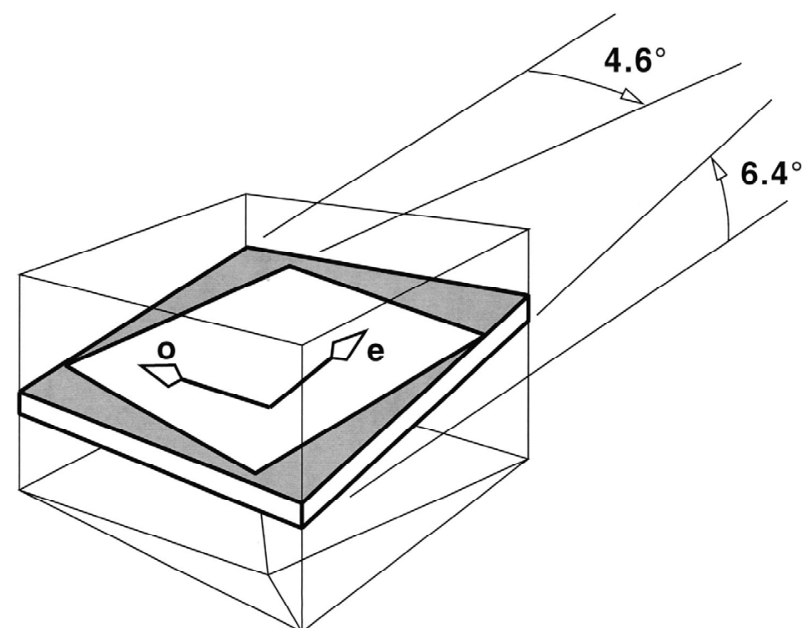

Figure 13. Diagram showing orientation of polarization smoothing plate inside the boule 


\section{CONCLUSION}

As the interface between the main laser and the target chamber, the NIF final optics is required to deliver high-level $3 \omega$ performance while maintaining diverse operational capability. A broad overview of the system has been presented with emphasis on the important functions of frequency conversion and beam conditioning. With the commissioning of the first NIF quad we have had the opportunity to test the integrated FOA design in its entirety. The coming year will continue to see improvements in the design as we go through a final value engineering cycle in preparation for full LRU production.

\section{REFERENCES}

1. J. Paisner and J. R. Murray, "Overview of the National Ignition Facility Project," Proc. SPIE Conf. Solid-State Laser Apps. To ICF (Monterey, CA, 1998).

2. P. J. Wegner, J. M. Auerbach, C.E. Barker, S.C. Burkhart, S.A. Couture, J.J. DeYoreo, R.L. Hibbard, L.W. Liou, M.A. Norton, P.K. Whitman and L.A. Hackel, "Frequency Converter Development for the National Ignition Facility," Proc. SPIE Conf. Solid-State Laser Apps. To ICF (Monterey, CA, 1998).

3. J.K. Lawson, D.M. Aikens, R.E. English Jr. and C.R. Wolfe, "Power spectral density specifications for highpower laser systems," Proc. Soc. Photo-Opt. Instrum. Eng., Vol. 2775, 345 (1996).

4. J.J. De Yoreo, A.K. Burnham, and P.K. Whitman, "Developing KH2PO4 and KD2PO4 crystals for the world's most powerful laser," International Materials Reviews, 47, 113-152 (2002).

5. C.E. Barker, R.A. Sacks, B.M. Van Wonterghem, J.A. Caird, J.R. Murray, J.H. Campbell, K.R. Kyle, R.B. Ehrlich, N.D. Nielsen, "Transverse stimulated Raman scattering in KDP," Proc. SPIE Conf. Solid-State Laser Apps. To ICF (Monterey, CA, 1995).

6. B.M. Van Wonterghem et al., "NIF commissioning and performance," paper this proceedings.

7. P.J. Wegner, B.M. Van Wonterghem, S.N. Dixit, M.A. Henesian, C.E. Barker, C.E. Thompson, L.G. Seppala and J.A. Caird, "Characterization of third-harmonic target plane irradiance on the National Ignition Facility Beamlet demonstration project," J. of Fusion Tech., 30, 539-547 (1996).

8. Y. Kato, K. Mima, N. Miyanaga, S. Arinaga, Y. Kitagawa, M. Nakatsuka and C. Yamanaka, "Random phasing of high-power lasers for uniform target acceleration and plasma instability suppression," Phys. Rev. Lett., 53, 10571060 (1984).

9. S.N. Dixit, M.C. Rushford, I.M. Thomas and M.D. Perry, "Continuous contour phase plates for tailoring the focal plane irradiance profile,” Proc. SPIE Conf. Solid-State Laser Apps. To ICF (Monterey, CA, 1995).

10. J. A. Menapace, S. N. Dixit, F. Y. Génin and W. F. Brocious, "Magnetorheological Finishing for Imprinting Continuous Phase Plate Structures onto Optical Surfaces", Laser Induced Damage in Optical Materials, Boulder Damage Symposium Proceedings (Boulder, Co, 2003).

11. K. Tsubakimoto, T. Jitsuno, N. Miyanaga, M. Nakatsuka, T. Kanabe and S. Nakai, Opt. Comm. 91, 9 (1992).

12. D. H. Munro, S.N. Dixit, A.B. Langdon and J.R. Murray, "Polarization smoothing in a convergent beam," submitted to Applied Optics, August 12, 2003. 